\title{
Increasing Trust in Meta-Heuristics by Using MAP-Elites
}

\author{
Neil Urquhart \\ School Of Computing, Edinburgh \\ Napier University \\ n.urquhart@napier.ac.uk
}

\author{
Michael Guckert \\ Technische Hochschule Mittelhessen \\ michael.guckert@mnd.thm.de
}

\author{
Simon Powers \\ School Of Computing, Edinburgh \\ Napier University \\ s.powers@napier.ac.uk
}

\begin{abstract}
Intelligent AI systems using approaches containing emergent elements often encounter acceptance problems. Results do not get sufficiently explained and the procedure itself can not be fully retraced because the flow of control is dependent on stochastic elements. Trust in such algorithms must be established so that users will accept results, without questioning whether the algorithm is sound. In this position paper we present an approach in which the user gets involved in the optimization procedure by letting them chose alternative solutions from a structure-archive which is created by the MAP-Elites algorithm. Analysis of these alternatives along the criteria of multiobjective optimization problems makes solutions comprehensible and hence is a means to build trust. We propose that the solution-focused nature of MAP-Elites allows the history of a solution to be easily shown to the user, explaining why that solution was included in those presented to the user. Here we demonstrate our ideas using a logistics problem previously explored by the authors.
\end{abstract}

\section{CCS CONCEPTS}

- Computing methodologies $\rightarrow$ Search methodologies; Discrete space search; $\bullet$ Applied computing $\rightarrow$ Transportation.

\section{KEYWORDS}

Vehicle Routing, Quality-Diversity Algorithms

\section{ACM Reference Format:}

Neil Urquhart, Michael Guckert, and Simon Powers. 2019. Increasing Trust in Meta-Heuristics by Using MAP-Elites. In Genetic and Evolutionary Computation Conference Companion (GECCO '19 Companion), July 13-17, 2019, Prague, Czech Republic. ACM, New York, NY, USA, 4 pages. https://doi.org/ $10.1145 / 3319619.3326816$

\section{INTRODUCTION}

\subsection{What is Trust?}

Complex intelligent systems that either contain elements in which they must first be prepared with examples (e.g. supervised learning in neural networks) or contain stochastic elements (e.g. evolutionary algorithms using stochastic operators) often meet acceptance problems as the way they compute their results is not transparent,

Permission to make digital or hard copies of all or part of this work for personal or classroom use is granted without fee provided that copies are not made or distributed for profit or commercial advantage and that copies bear this notice and the full citation on the first page. Copyrights for components of this work owned by others than ACM must be honored. Abstracting with credit is permitted. To copy otherwise, or republish, to post on servers or to redistribute to lists, requires prior specific permission and/or a fee. Request permissions from permissions@acm.org.

GECCO '19 Companion, July 13-17, 2019, Prague, Czech Republic

(c) 2019 Association for Computing Machinery.

ACM ISBN 978-1-4503-6748-6/19/07...\$15.00

https://doi.org/10.1145/3319619.3326816 and a notion that they were achieved fortuitously persists for the non expert. For example, an evolutionary algorithm may search through millions of possible solutions, using operators such as recombination and mutation to move between solutions. This can hardly be retraced without appropriate tools. Another example are algorithms that are trained on examples and therefore highly depend on the quality of the input data, and any bias within it. Because of elements that make use of emergent structures, reproduction of decisions and retracing the algorithm ex post is difficult. Users applying such systems may be left in doubt about quality and validity of the results and are reluctant to accept them. Andras et al have outlined the idea that trust in intelligent machines can be achieved by repeated, successful use of a system (called inductive trust) [3]. This trust building process can be initiated by establishing mechanisms in which the results are explained and justified in a form that is accessible to human beings. Various approaches to making such methods more explainable have been presented. Saving meta-information during the execution of the algorithm may facilitate the construction of an explanation of the final solution. For example, neural networks may highlight data that was most influential on the produced output and which was not [9]. Analogously, for population based approaches, an explanation that traces the evolution of the chosen solution may provide some form of justification for the final solution.

\subsection{MAP-Elites}

The Multi-dimensional Archive of Phenotypic Elites (MAP-Elites) is an illumination algorithm that was first introduced by Mouret et al [7]. MAP-Elites creates a structure archive of high-performing solutions mapped onto solution characteristics defined by the user. A set of characteristics is identified which may be used to classify a solution (e.g. for a routing problem one might use cost, distance, delivery time span and vehicles required). Solutions are generated using mutation and recombination operators, but each solution can be classified by normalizing its characteristics in order to identify a "bin" within the solution space that the solution belongs to. For instance we might normalize our four characteristics on a scale of 020 , thus a solution might occupy a bin such as 5:4:2:12 for example. The number of bins in a map is calculated as $s^{d}$ where $s$ is the number of points on the scale and $d$ is the number of dimensions. In our example the number of bins would be $160,000\left(20^{4}\right)$. There exists the issue as to what happens when a solution is generated that belongs to a bin that is already occupied. In this case MAPElites uses a fitness value to determine which solution should be allowed to occupy the bin. In our vehicle routing problem (VRP) example we could utilize distance as the fitness value and thus, when a solution is found that maps to an occupied bin, it replaces the existing solution if it represents a decrease in distance. Figure 1 
shows a representation of an archive as produced by MAP-Elites based on a 4-dimensional problem as described above.

MAP-Elites provides the user with a structured set of solutions from which they may make the choice of final solution.

\subsection{The Micro-Depot Problem}

In this paper we use an urban logistics problem as an example problem domain, this problem is more fully explored by the authors in [8]. The Micro-Depot Problem (MDP) is a VRP problem, but with the addition of micro-depots which are used to transfer goods for final delivery using walking couriers or cargo bikes. Parcels are delivered to the micro-depots by a supply-vehicle from a central depot. Parcels can also be delivered to customers directly by the supply-vehicle itself rather than being dropped at a depot. We characterize solutions to the micro-depot problem as follows:

- Pollution: attempt to improve air-quality in the city through lowering emissions.

- Distance: reduce congestion by reducing the overall distance travelled.

- Couriers: the number of couriers required to implement the solution.

- Time: the timespan required to make all of the deliveries

In addition the financial cost of the solution is used as the fitness function, financial cost being the sum of the fixed costs and costs $/ \mathrm{km}$ for the couriers used in the solution. The problem may be solved using MAP-Elites based on the four dimensions of pollution $\left(\mathrm{CO}_{2}\right)$, distance $(\mathrm{km})$ and couriers are all normalized on a scale of 0-20.

\section{SOLVING THE MICRO-DEPOT PROBLEM}

We use an representation and operators that are fully described by the authors in [8]. The representation uses a grand tour which represents the route to be taken using the supply vehicle to visit all customers, this route is constructed using the nearest-neighbour heuristic. A chromosome is which contains instructions to transfer groups of deliveries to a micro-depot. Each gene within the chromosome represents one particular courier as follows:

- Tour point

- Customer qty

- Depot ID

- Courier mode

An example gene might be : 5, 3, D1, WALK which would remove the customers at positions 5,6 and 7 in the grand tour and have them delivered by a walking courier based at depot $\mathrm{d} 1$ (the 3 items removed from the grand tour are replaced by a single visit to d1). Mutation operators create new genes, delete old genes and randomly alter the contents of a selected gene. The recombination operator creates new chromosomes by randomly selecting genes from the parents.

In summary the chromosome is a set of instructions that may be used to convert the grand tour into a 2-tier delivery solution making using of micro-depots and couriers

\section{RECORDING ALGORITHMIC DECISIONS}

Evolutionary Algorithms and other stochastic Meta-Heuristics have traditionally had difficulties in inspiring trust in end-users. Typically an end-user (e.g. a domain expert such as a planner or scheduler) has an understanding of the solution, but not of the method used to create it. When the solution generated does not match the user's expectation they look for an explanation as to how that solution was created. A "traditional" EA generates solutions by means of recombination and mutation, both of which contain random elements. Solutions are then potentially incorporated within the population using a replacement strategy[5]. The random variations and chance of survival underpin the evolutionary search process. These are difficult to justify, but they are the means by which success is achieved. Explaining this process to an end-user can therefore present difficulties. Essentially, an end solution is the result of a number of random changes (mutations) and recombinations, each of which resulted in a change to the solution that improved its fitness. Attempts have been made to visualize the output of EAs [4], by tracking solutions and showing their antecedents. It can be argued that the stochastic nature of EAs makes such visualizations difficult to follow and of limited value when trying to explain how the characteristics of the final solution were determined.

It may be argued that MAP-Elites is solution spaced focus, as the archive is structured around solution characteristics. It then becomes possible to "explain" the history of a given bin to the user. Table 1 gives an example of the history of one specific bin. For example, if we assume that the user is interested in a solution with the characteristics 20:20:9:18, but wishes to know more about the history of the solution, then MAP-Elites can be used to produce a timeline. Table 1 shows that 13 solutions have occupied the cell. If we examine the final change we note that the number of couriers does not change but the emissions drop and the distance drops fractionally, whilst the overall time increases. We can surmise that this change is most likely a change in courier type to a lower emissions courier for a delivery run which results in less $\mathrm{CO}_{2}$, but takes slightly longer.

Table 1 also shows the origin of each individual placed into the bin, where two other bins are referenced then a crossover operator, followed by a mutation was used, where only 1 bin is referenced then the new solution was created by cloning followed by a mutation.

The ability to present a linear timeline as shown in table 1 is a major advantage when explaining how MAP-Elites has arrived at a given solution.

\section{INCREASING TRUST BY PRESENTING ALTERNATIVES}

A common complaint from end users presented with a solution that has been constructed using a meta-heuristic is that they have had no role in the solution construction. One manner in which endusers may be involved is by selecting the final choice of solution from those presented in the archive of elites. It could be argued that MAP-Elites is a solution space filter - it takes a solution space that is too large to be feasible for the user to comprehend, and filters out a structured set of solutions that are representative of the solution space. The total size of the map (assuming all bins are filled) is $20^{4}=$ 160000. It may therefore be argued that MAP-Elites is simply taking a massive search space that is impractical to search, and replacing it with a smaller search space that is still, from a user's perspective, 


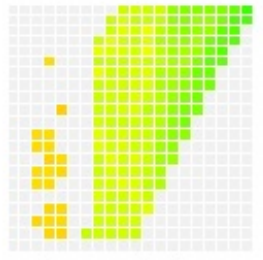

time:emissions

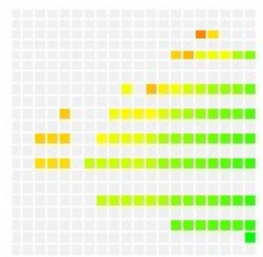

vehicles:emissions

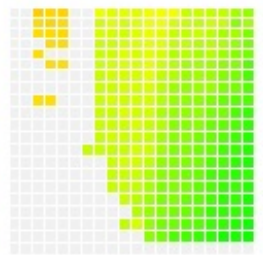

dist:emissions

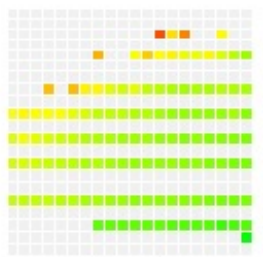

vehicles:time

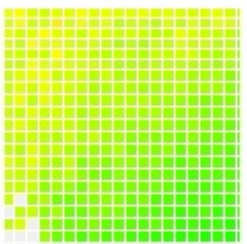

dist:time

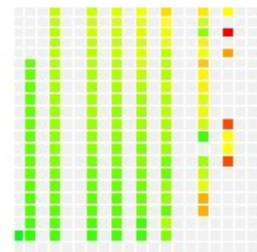

dist:vehicles

Figure 1: A visualisation of a 4-dimensional elite archive, shown as a set of $2 \mathrm{D}$ heat maps, each map shows the archive from the perspective of 2 dimensions, each pair of dimensions being covered. Each individual square within each map represents a "bin", if it is gray no solution has been found, if it is coloured then a solution has been found, the colour is based on the fitness of the solution occupying the bin.

\begin{tabular}{cccccccc}
\hline \multirow{2}{*}{ Time } & \multirow{2}{*}{ Origin } & \multirow{2}{*}{ Updates } & \multirow{2}{*}{ Fitness(cost) } & \multicolumn{4}{c}{ Solution Characteristics } \\
\cline { 5 - 7 } & & & & Couriers & Emissions & Distance & Time \\
\hline 5704 & $12: 4: 9: 11 / 12: 12: 4: 17$ & 0 & 977.11 & 5 & 45.48 & 21.25 & 11.66 \\
5779 & $17: 16: 9: 19 / 19: 20: 7: 9$ & 1 & 930.21 & 5 & 48.15 & 21.04 & 12.106 \\
14252 & $17: 16: 11: 18$ & 2 & 922.87 & 5 & 42.97 & 21.19 & 10.55 \\
17036 & $20: 20: 9: 18$ & 3 & 922.01 & 5 & 42.59 & 21.14 & 10.55 \\
50723 & $11: 16: 9: 18 / 12: 8: 7: 11$ & 4 & 919.24 & 5 & 40.92 & 21.10 & 10.61 \\
55009 & $11: 10: 9: 5 / 19: 17: 7: 15$ & 5 & 881.60 & 5 & 48.70 & 21.15 & 11.41 \\
124016 & $13: 12: 9: 7 / 18: 17: 9: 4$ & 6 & 880.66 & 5 & 47.90 & 21.21 & 12.24 \\
130454 & $15: 15: 11: 18 / 14: 7: 7: 19$ & 7 & 878.25 & 5 & 46.12 & 21.28 & 10.77 \\
207786 & $19: 19: 11: 12 / 15: 12: 11: 10$ & 8 & 878.22 & 5 & 47.49 & 20.85 & 11.1 \\
229666 & $10: 16: 7: 6$ & 9 & 877.06 & 5 & 46.58 & 20.90 & 11.06 \\
417443 & $14: 13: 13: 16 / 16: 15: 7: 16$ & 10 & 875.85 & 5 & 44.97 & 21.16 & 10.54 \\
863310 & $18: 18: 4: 2$ & 11 & 875.32 & 5 & 45.53 & 20.88 & 10.67 \\
1195610 & $20: 20: 7: 20$ & 12 & 874.23 & 5 & 44.88 & 20.87 & 11.00 \\
\hline
\end{tabular}

Table 1: The "history" of a specific bin (20:20:9:18) within the archive. Each row represents an update of the cell contents, at each point the fitness improves. The details of the change made may be ascertained by examining the solution characteristics.

difficult to navigate. In order to increase trust in MAP-Elites we need to support the end user when they are making the final choice of solution. The end user may be supported by visualizing the map of solutions using a technique such as Parallel Coordinate (PC). Within a Parallel Coordinate plot solutions are represented as a Polyline that intersects vertical axis at the appropriate points, for a full description of the technique the reader is referred to [6]. A PC plot of the output from a typical run of MAP-Elites may be seen in figure 2. In order for the plot to support the user it is necessary that the user interacts with the plot, tools to support such interaction include PACO [2] and ElVis [1]. Such tools allow interaction with the PC plot, as shown in figure 3 which demonstrates the user having highlighted areas of interest on the 2nd and 3rd axis (time and vehicles) leading to only those solutions that match the users' criterion being highlighted. In this way the user may be guided through the the solutions found and be able to visualize alternatives as they are supported towards making their the final choice.

\section{CONCLUSIONS AND FUTURE WORK}

Here we have presented an argument that MAP-Elites can help to explain the operation of stochastic population-based optimization algorithms to users. To illustrate this, we used an example of a vehicle routing problem, where MAP-Elites was used to construct a set of solutions in the context of the criteria of a multi-objective optimization problem. We enrich the solution archive with a time line showing how the solutions were arrived at. We hypothesize that this, in turn, can help to increase trust in algorithms with stochastic elements, or that depend on potentially biased training data. The next step is to empirically test this hypothesis, through experiments involving a sufficiently large number of domain experts who do not have knowledge of the underlying algorithms. This could be done by a split test involving showing half of the sample of users the MAP-Elites visualization, and the other half a standard visualization of a Pareto front, for example.

We also propose that trust by induction may be engendered in users through the use of tools such as those described in section 4, in particular the ElVis tool ([1]) which allows the user to to interact with the parallel coordinates plot and to see a visualisation of each solution within the plot.

\section{REFERENCES}

[1] [n. d.]. ElVis - Elite Visualisations. http://https://commute.napier.ac.uk/upload/ [2] [n.d.]. parallelcoordinates.de. http://www.parallelcoordinates.de/ 


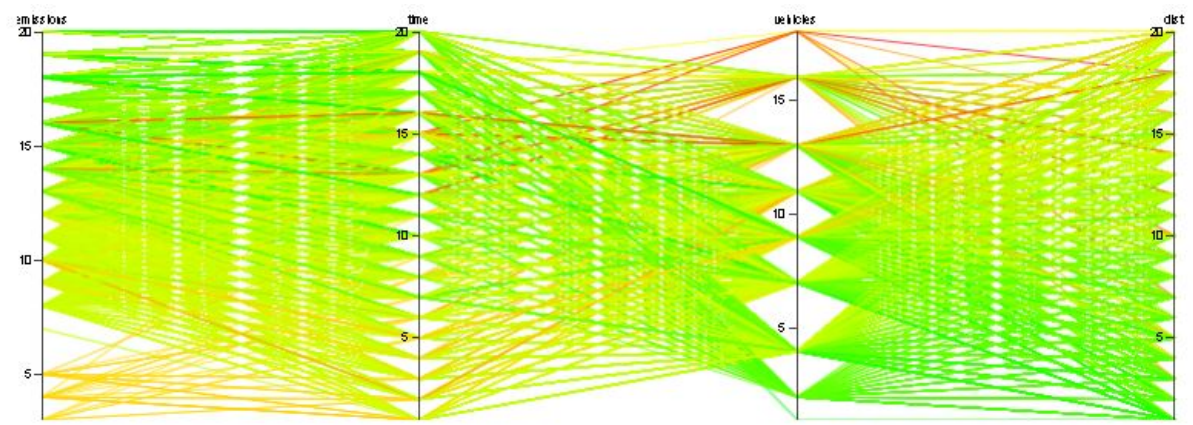

Figure 2: A Parallel Coordinate plot of all the solutions returned from MAP-Elites.

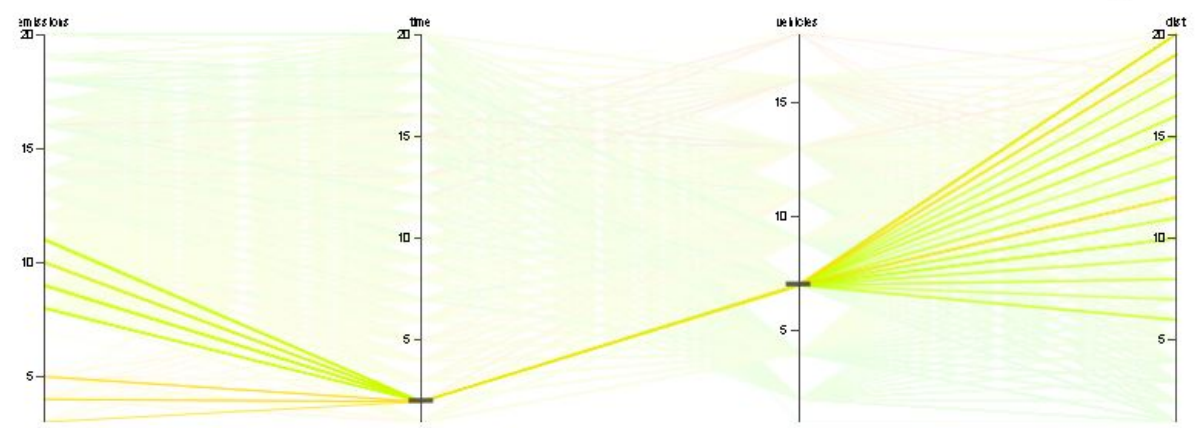

Figure 3: The plot shown in figure 2, but with the user specifying criterion on two axis in order to highlight specific solutions.

[3] L. Andras, P. snd Esterle, M. Guckert, T. Han, P. Lewis, K. Milanovic, T. Payne C. Perret, J. Pitt, S. Powers, N. Urquhart, and S. Wells. 2018. Trusting Intelligent Machines. IEEE Technology and Society Magazine 37, 4 (2018), 76âAŞ83. https: //doi.org/10.1109/MTS.2018.2876107

[4] E. Hart and P. Ross. 2001. GAVEL - a new tool for genetic algorithm visualization IEEE Transactions on Evolutionary Computation 5, 4 (2001), 335âĂŞ348. https: //doi.org/10.1109/4235.942528

[5] John H. Holland. 1992. ADAPTATION IN NATURAL AND ARTIFICIAL SYSTEMS: an Introductory Analysis with Applications to Biology, Control, and Artificial Intelligence. The MIT Press.
[6] Alfred Inselberg and Ben Shneiderman. 2009. Parallel coordinates: visual multidimensional geometry and its applications. Springer.

[7] Jean-Baptiste Mouret and Jeff Clune. 2015. Illuminating search spaces by mapping elites. CoRR (2015).

[8] Silke Hoehl Neil Urquhart and Emma Hart. 2019. An Illumination Algorithm Approach to Solving the Micro-Depot Routing Problem. Proceedings of the Genetic and Evolutionary Algorithms Conference (2019).

[9] Wojciech Samek, Thomas Wiegand, and Klaus-Robert Müller. 2017. Explainable Artificial Intelligence: Understanding, Visualizing and Interpreting Deep Learning Models. CoRR abs/1708.08296 (2017). arXiv:1708.08296 http://arxiv.org/abs/1708. 08296 